\title{
CLASSIFICATION OF UPPER AIRWAYS IMAGES FOR ENDOTRACHEAL INTUBATION VERIFICATION
}

\author{
Dror Lederman \\ Holon Institute of Technology, Holon, Israel \\ drorlehit.ac.il
}

\begin{abstract}
This paper addresses the problem of classification of upper airways images for endotracheal intubation verification in order to improve the safety of patients undergoing general anaesthesia. The proposed method is based on textural features utilized in a continuous probabilistic framework using parallel Gaussian mixture models (GMMs). The classification decision is made based on a maximum likelihood approach, which is insensitive to the angle at which the image was taken. Evaluation of the proposed approach is done using a dataset of 200 images that includes three classes of anatomical structures of the upper airways. The results show that the approach can be used to efficiently and reliably represent and classify medical images acquired during various procedures.
\end{abstract}

\section{KEYWORDS}

Artificial intelligence, intubation verification, Gaussian mixture models, textural features.

\section{INTRODUCTION}

Intubation is performed widely in hospitals and emergency medical units. During intubation, a flexible tube is used to secure passage of air to and from the lungs. The procedure is performed by manually opening the mouth, lifting the tongue using a device called laryngoscope in order to reveal the vocal cords, and inserting an endotracheal tube (ETT) through the vocal cords. The ETT should be positioned between 2 and $5 \mathrm{~cm}$ above the bifurcation of the trachea into the two primary bronchi ("carina").

The anatomy of the patient does not always allow easy insertion of the ETT and consequently it might be incorrectly positioned, usually either in the esophagus or in the right main bronchus. Both of these conditions can produce catastrophic results, as the patient might be deprived of oxygen. Unintentional esophageal intubation has been associated with high mortality rate $[1,2]$. In cases of right lung intubation (also termed one-lung intubation (OLI)), only one lung is ventilated. Prolonged one lung ventilation might cause serious pulmonary complications such as collapse of the contralateral lung and hyperinflation of the ventilated lung, which might eventually result in hypoxia and pneumothorax, respectively, and has been associated with a significant increase in morbidity [3,4] and Pneumonia [5]. Both esophageal and OLI may occur after the ETT was positioned correctly ("dislodgement") from many reasons, for example, due to neck flexion during general anesthesia $[6,7]$.

David C. Wyld et al. (Eds) : CSITA, ISPR, ARIN, DMAP, CCSIT, AISC, SIPP, PDCTA, SOEN - 2017

pp. 77-83, 2017. (C) CS \& IT-CSCP 2017

DOI : $10.5121 /$ csit.2017.70108 
Confirmation of correct tube positioning is a challenging task that requires high skills and the use of secondary objective devices. Numerous studies, which investigated endotracheal misplacement rates in hospital and pre-hospital settings, reported rates between $0 \%$ and $25 \%$, depending among others, on study design [1, 2, 5, 8-13].

There are various methods and techniques for endotracheal intubation confirmation. The most common technique is auscultation to lung sounds using a stethoscope. This technique requires high attention, and its reliability has been questioned in many studies [14-20]. The use of exhaled carbon dioxide detection (CO2) measurements (termed end-tidal CO2 (ETCO2)), has become the gold standard-de-facto for confirming correct tube positioning. However, the method has been found to be unreliable in many emergencies [21-25]. In addition, the method can not be used to detect OLI incidents as in such cases the capnogram is generally typical in shape and shows normal ETCO2 values [22, 25]. Other techniques have been proposed (e.g., [26-30]), but none of them has been proven effective. Therefore, attempts to find the ultimate technique for correct tube position confirmation have been continued.

In this paper, we further develop a previously-proposed system [31, 32] for endotracheal intubation confirmation. The system is based on identification of specific anatomical landmarks as indicators of correct or incorrect tube positioning, based on a continuous probabilistic approach using textural features.

\section{MATERIAL AND METHODS}

\subsection{The System}

Intubation is usually performed using an intubating stylet, used to control and guide the ETT. We designed and assembled a designated video-stylet. The tip of the stylet comprises a miniature complementary metal oxide silicon (CMOS) sensor. The inner part of the stylet contains wires to transfer the image and a narrow lumen to spray water or air in order to clear blood and secretions away from the camera sensor. The image sensor is connected to a processor with an integrated image acquisition component. During intubation, this rigid stylet is inserted into a standard ETT with its camera at the tip. Video signals are continuously acquired and processed by the confirmation algorithm implemented on the processor.

\subsection{Pre-Processing and Textural Features}

The classification system is a supervised one and comprises three main steps. The first step is conversion of the acquired image from a color image (RGB) to a grey scale image, which is followed by feature extraction. Various features have been utilized in medical image applications, including squared grey-level difference [33], cross correlation [33] and localized intensity features [34]. In this work, textural features [35, 36] were used. Textural features contain important information about the structural arrangement of surfaces and their relationship to the surrounding environment. In particular, features based on grey level co-occurrence matrices (GLCM) were utilized. These features are based on the assumption that textural information on an image is contained in the overall or "average" spatial relationship, which the grey tones in the image have to one another [37]. More specifically, it is assumed that this textural information is adequately specified by a set of grey tone spatial dependence matrices which are computed for various angular relationships and distances between neighbouring resolution cell pairs on the image. The advantages of these features is that they are robust to small differences in the imaging directions and scaling. This property is of great importance in many medical imaging applications. 


\subsection{The Gaussian Mixture Model (GMM) Probabilistic Framework}

In order to classify the images, a probabilistic framework is utilized, in which the images are represented in the feature space using Gaussian mixture models (GMMs). GMM based classification methods have been largely applied to speech recognition [38], and recently to some medical image categorization applications [31, 34]. Mixture models, in particular GMM, form a common technique for probability density estimation. This is justified by the fact that any density can be estimated, in a required degree of approximation, using finite Gaussian mixture [39]. Their mathematical properties, as well as their flexibility and the availability of efficient estimation algorithms, make them attractive for classification problems. The most popular algorithm for GMM parameters estimation is the expectation-maximization (EM) [39]. This algorithm allows iterative optimization of the mixture parameters, under monotonic likelihood requirements, and has a relatively simple implementation. In this study, the probability density functions (pdf) of the images were represented, in the feature space, by three classes, where each class was modeled by a combination of four GMMs. Each GMM, representing a random process, $x$, is defined as a weighted sum of K Gaussian components, as follows:

$$
f_{K}(\mathbf{x})=\sum_{k=1}^{K} \pi_{k} \phi_{\theta_{k}}(\mathbf{x}),
$$

where $\pi_{k}$ represents the mixing weight such that $\sum_{k=1}^{K} \pi_{k}=1, \pi_{j} \geq 0 \forall j$ and $\phi\left(\mathbf{x} ; \theta_{j}\right)$ represents the $j^{\text {th }}$ d-dimensional Gaussian mixture component given by:

$$
\begin{aligned}
& \phi\left(\mathbf{x} ; \theta_{j}\right)=(2 \pi)^{-d / 2}\left|\mathbf{S}_{j}\right|^{-1 / 2} \\
& \exp \left[-0.5\left(\mathbf{x}-\mathbf{m}_{j}\right)^{T} \mathbf{S}_{j}^{-1}\left(\mathbf{x}-\mathbf{m}_{j}\right)\right]
\end{aligned},
$$

which is parameterized on the mean $\mathbf{m}_{j}$ and the covariance matrix $\mathbf{S}_{j}$, collectively denoted by the parameter vector $\theta_{j}$.

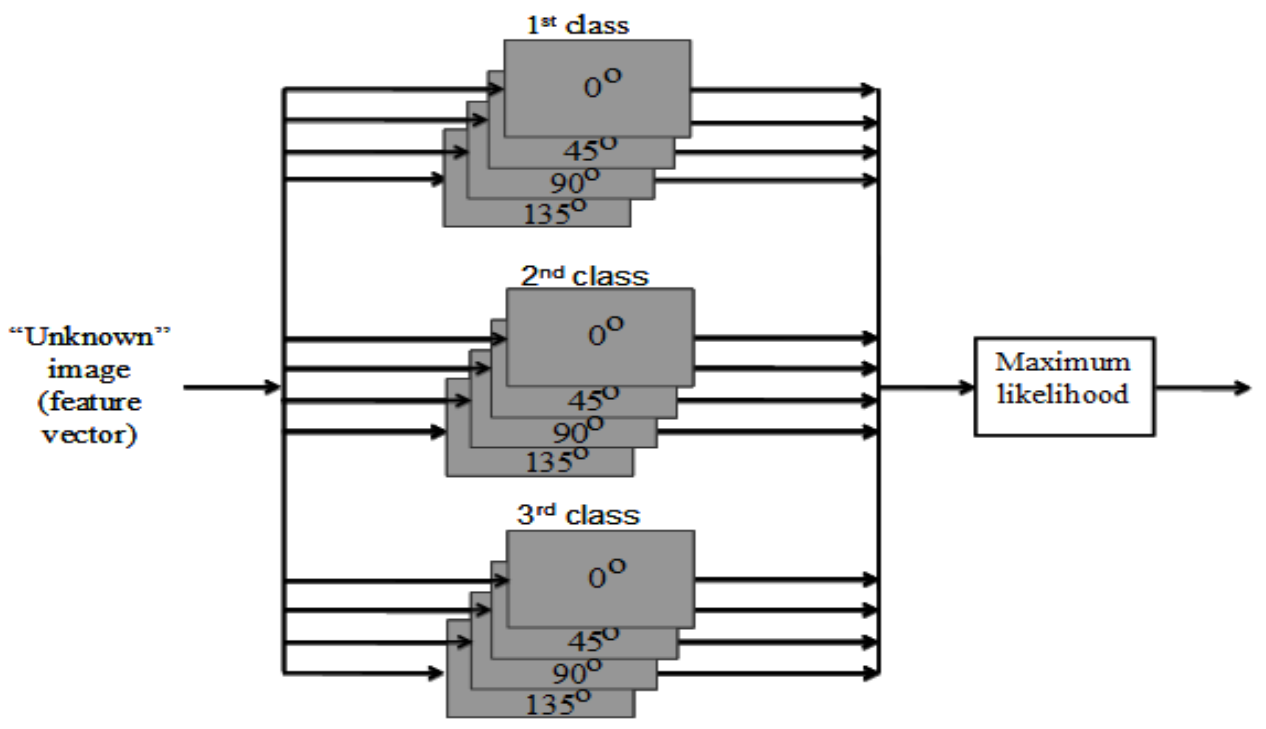

Figure 1. The parallel model approach 


\subsection{The Parallel GMMs Scheme}

During endotracheal intubation, a common procedure in operating rooms, the tube is inserted into the patient's mouth in an horizontal direction, i.e., an angle of $0^{\circ}$, where the head of the tube turns away from the patient. While inserting the tube into the trachea, the medical professional rotates the tube counter clockwise until it reaches a vertical direction $\left(90^{\circ}\right)$. Therefore, the acquired images might be rotated with respect to the images that were used to train the models and therefore pre-alignment of the images prior to their classification is required. Traditionally, two approaches have been used to overcome this common problem: (a) employ an image registration technique prior to classification [40], (b) utilize features which are insensitive to the imaging angle [37]. Common to these approaches is their computational complexity, which is a major drawback when real-time classification is required. An alternative model based approach is proposed, according to which several models are used to represent each class of images, where each "sub-model" represents images taken at a specific range of angles, i.e., a GMM for angles around $0^{\circ}$, a GMM for angles around $45^{\circ}$, etc. During the training phase, a model is estimated using the images of each and every class, resulting in a total of 12 models (4 models for each one of the classes). During the testing phase, an "unknown" image is classified according to a generalized maximum likelihood rule, i.e., the selected model is the one which one of its submodels provides the maximum likelihood (ML). The classification decision rule is therefore given by:

$$
m=\underset{l}{\arg }\left\{\max _{l=1,2 ; s=1,2, \ldots, S} f\left(O \mid \lambda_{l s}\right)\right\},
$$

where $\lambda_{l s}$ represents the $s$ th sub-model of the $l$ th group. Figure 1 presents the parallel models scheme.

\section{RESULTS}

In order to evaluate system performance, we used a database of 200 images recorded from the upper airways of 10 patients where the images were taken at different angles and directions. The details of this database appear elsewhere [41]. The dataset used in this work includes 200 randomly-chosen images from the available database, that were visually inspected by a medical expert and classified into one of the following categories: upper-trachea, carina and esophagus. Each of these classes was further divided into four sub-groups representing 4 quantized imaging angles- $0^{\circ}, 45^{\circ}, 90^{\circ}$ and $135^{\circ}$. Evaluation of the proposed approach was performed using a leave-one-out validation method: in each iteration, 199 of the images were used to train the models and the remaining image was used to test system performance. This process was repeated 200 times, such that each image participated once in the testing phase.

The classification results are summarized in Table 1, where the rows represent the predicted (identified) classes and the columns represent the actual classes. In this case, the order of each one of the GMMs was set to 8 . The system achieved an overall classification rate of $92.0 \%$.

Table 1. Confusion matrix of the three classes

\begin{tabular}{|c|c|c|c|}
\hline \multirow{2}{*}{ Identified } & \multicolumn{3}{|c|}{ True } \\
\cline { 2 - 4 } & Upper tracheal & Carina & Esophagus \\
\hline Upper tracheal & $92 \%$ & $3 \%$ & $5 \%$ \\
\hline Carina & $5 \%$ & $94 \%$ & $6 \%$ \\
\hline Esophagus & $3 \%$ & $3 \%$ & $89 \%$ \\
\hline
\end{tabular}




\section{CONCLUSiOnS}

A method for classification of upper airways images based on a probabilistic framework using textural features was presented. A parallel scheme utilizing GMMs is used to represent images taken at different angles. While the training phase, which is usually a one-time phase, requires a tedious manual segmentation of the dataset according to the different sub-groups (for each range of imaging angles), the testing phase is based on a simple ML decision rule among the models of each class. Therefore, the proposed scheme is simple and computationally efficient. It should be noted however that further work is needed in order to evaluate the performance of the proposed approach and to compare it with other methods proposed in the literature.

All of the algorithms used in this work were implemented in Matlab R2016a 64bit. Using a conventional PC equipped with Dual Intel Xeon $3.4 \mathrm{GHz}$ with a 4 GBytes of RAM, feature extraction requires about 1.5 second for each image and the parallel ML based decision rule requires approximately 1 second for each image. Efforts are currently being invested to collect a larger database in order to allow a more thorough investigation of the proposed approach.

\section{REFERENCES}

[1] S. Silvestri, G. A. Ralls, and B. Krauss, "The effectiveness of out-of-hospital use of continuous endtidal carbon dioxide monitoring on the rate of unrecognized misplaced intubation within a regional emergency medical services system," Ann. Emerg. Med., vol. 45, pp. 497-503, 2005.

[2] A. Timmermann, S. G. Russo, C. Eich, M. Roessler, U. Braun, W. H. Rosenblatt, and M. Quintel, "The out-of-hospital esophageal and endobronchial intubations performed by emergency physicians," Crit. Care and Trauma, vol. 104, pp. 619-623, 2007.

[3] R. L. Owen and F. W. Cheney, "Endobronchial intubation: a preventable complication," Anesthesiology, vol. 67, pp. 255-257, 1987.

[4] C. W. Zwillich, D. J. Pierson, and C. E. Creagh, "Complications of assisted ventilation, a prospective study of 354 consecutive episodes," Am. J. Med., vol. 57, pp. 161-170, 1974.

[5] H. E. Wang, L. J. Cook, C. H. Chang, D. M. Yealy, and J. R. Lave, "Outcomes after out-of-hospital endotracheal intubation errors," Resuscitation, vol. 80, pp. 50-55, 2009.

[6] S. T. Vergese, R. S. Hannallah, M. C. Slack, R. R. Cross, and K. M. Patel, "Auscultation of bilateral breath sounds does not rule out endobronchial intubation in children," Anesth. Analg., vol. 56-58, 2004.

[7] S. J. Yap, R. W. Morris, and D. A. Pybus, "Alterations in endotracheal tube position during general anesthesia," Anaesth. Crit. Care, vol. 586-588, 1994.

[8] L. M. Jacobs, L. D. Berrizbeitia, B. Bernnett, and C. Madigan, "Endotracheal intubation in the prehospital phase of emergency medical care," JAMA, vol. 250, 1983.

[9] M. E. Jemmet, K. M. Kendal, and M. W. Fourre, "Unrecognized misplacement of endotracheal tubes in a mixed urban to rural emergency medical services setting," Acad. Emerg. Med., vol. 10, pp. 961965, 2003.

[10] J. H. Jones, M. P. Murphy, and R. L. Dickson, "Emergency physician-verified out-of-hospital intubation: miss rates by paramedics," Acad. Emerg. Med., vol. 11, pp. 707-709, 2004.

[11] S. H. Katz and J. L. Falk, "Misplaced endotracheal tubes by paramedics in an urban emergency medical services system," Ann. Emerg. Med., vol. 37, pp. 32-37, 2001. 
[12] J. E. Pointer, "Clinical characteristics of paramedics' performance of endotracheal intubation," J. Emerg. Med., vol. 6, 1988.

[13] R. D. Steward, P. M. Paris, and P. M. Winter, "Field endotracheal intubation by paramedical personnel: success rates and complications," Chest, vol. 85, 1984.

[14] W. Brunel, D. L. Coleman, and D. E. Schwartz, "Assessment of routine chest reoentgenograms and the physical examination to confirm endotracheal tube position," Chest, vol. 96, pp. 1043-1045, 1989.

[15] T. H. Howells, "Oesophageal misplacement of a tracheal tube," Anaesthesia, vol. 40, pp. 398-389, 1985.

[16] I. D. Klepper, R. K. Webb, J. V. D. Walt, G. L. Ludbrooks, and J. Cockings, "The sthethoscope: Application and limintation- an analysis of 2000 incidents reports," Anaesth. Intens. Care, vol. 21, pp. 575-578, 1993.

[17] K. Linko, M. Paloheimo, and T. Tammisto, "Capnograhphy for detection of accidental oesophageal intubation," Acta Anaesthesiol. Scand., vol. 27, pp. 199-202, 1983.

[18] A. W. Peterson and L. M. Jacker, "Death following inadvertent esophageal intubation: a case report," Anesth. Analg., vol. 52, pp. 398-401, 1973.

[19] H. E. Wang, J. R. Lave, C. A. Sirion, and M. Yealy, "Paramedic intubation errors: isolated events or symptoms of larger problems?," Health Affairs, vol. 25, pp. 501-509, 2006.

[20] G. R. Wodicka, P. D. DeFrain, and S. S. Kraman, "Bilateral asymmetry of respiratory acoustic transmission," Med. Biol. Eng. Comp., vol. 32, pp. 489-494, 1994.

[21] S. M. Bhende and A. E. Thompson, "Evaluation of an end-tidal CO2 detector during pediatric cardiopulmonary resuscitation," Pediat., vol. 95, pp. 395-399, 1995.

[22] J. S. Gravenstein, M. B. Jaffe, and D. A. Paulus, Capnograhpy clinical aspects: Cambridge University Press, 2004.

[23] J. Li, "Capnography alone is imperfect for endotracheal tube placement confirmation during emergency intubation," J. Emerg. Med., vol. 20, pp. 223-229, 2001.

[24] J. P. Nolan, C. D. Deakin, and J. Soar, "European resuscitation council guidelines for resuscitation," Resuscitation, vol. 67, pp. S39-S86, 2005.

[25] R. K. Webb, J. H. V. D. Walt, W. B. Runciman, J. A. Williamson, J. Cockings, W. J. Russel, and S. Helps, "Which monitor? an analysis of 2000 indicent reports," Anaesth. Intens. Care, vol. 21, pp. 529$542,1993$.

[26] D. Lederman, "An energy ratio test for one lung intubation detection," in 18th Biennial International EURASIP conference, Brno, Czech Republic, 2006.

[27] C. J. O'connor, H. Mansy, R. A. Balk, K. J. Tuman, and R. H. Sandler, "Identification of endotracheal tube malpopsitions using computerized analysis of breath sounds via electronic stethoscopes," Anesth. Analg., vol. 101, pp. 735-739, 2005.

[28] S. Tejman-Yarden, D. Lederman, N. Weksler, and G. Gurman, "Acoustic monitoring of double lumen ventilated lungs for the detection of selective unilateral lung ventilation," Anesth. Analg., vol. 103, pp. 1489-1492, 2006.

[29] S. Tejman-Yarden, A. Zlotnik, L. Weizman, J. Tabrikian, A. Cohen, N. Weksler, and G. M. Gurman, "Acoustic monitoring of lung sounds for the detection of one-lung intubation," Anesth. Analg., vol. 105, pp. 397-404, 2007. 
[30] L. Weizman, J. Tabrikian, and A. Cohen, "Detection of one-lung intubation incidents," Annals of Biomed. Eng., vol. 36, pp. 1844-1855, 2008.

[31] D. Lederman, "An endotracheal intubation confirmation system based on carina image detection- a preliminary assessment," Med. \& Biol. Eng. \& Comp., vol. 49(1), pp. 75-83, 2011.

[32] D. Lederman, "Endotracheal intubation confirmation based on video image classification using a parallel GMMs framework- a preliminary evaluation," Annals of Biomed. Eng., vol. 39(1), pp. 508$513,2011$.

[33] D. Deguchi, K. Mori, M. Feuerstein, T. Kitasaka, C. R. Maurer, Y. Suenaga, H. Takabatake, M. Mori, and H. Natori, "Selective image similarity measure for bronchoscope tracking based on image registration," Medical Image Analysis, vol. 13, pp. 621-633, 2009.

[34] H. Greenspan and A. T. Pinhas, "Medical image categorization and retrieval for PACS using the GMM-KL framework," IEEE Trans. on Inform. Tech. in Biomed., vol. 11, pp. 190-202, 2007.

[35] G. Castellano, L. Bonilha, L. M. Li, and F. Cendes, "Texture analysis of medical images," Clinical Radiology, vol. 59, pp. 1061-1069, 2004.

[36] J. Tan, B. Zheng, W. Wang, D. Lederman, J. Pu, F. C. Sciurba, D. Gur, and J. K. Leader, "Texturebased segmentation and analysis of emphysema depicted on CT images," presented at the Proc SPIE, 2011.

[37] R. M. Haralick, M. Shanmugam, and I. Dinstein, "Textural features for image classification," IEEE Trans. on Systems, Man, and Cybernatics, vol. SMC-3, pp. 610-621, 1973.

[38] R. C. Rose and D. A. Reynolds, "Text-independent speaker identification using automatic acoustic segmentation," presented at the IEEE Int. Conf. Acoust., Speech and Signal Proc., 1990.

[39] A. P. Dempster, N. M. Laird, and D. B. Rubin, "Maximum likelihood from incomplete data via the EM algorithm," J. Royal Stat. soc., vol. 39, pp. 1-38, 1977.

[40] Y. Keller, Y. Shkolnisky, and A. Averbuch, "The Angular Difference Function and its application to image registration," IEEE Trans. Pattern Analysis and Machine Intelligence, vol. 27, pp. 969-976, 2005 .

[41] D. Lederman, S. Lampotang, and M. Shamir, "Automatic endotracheal tube position confirmation system based on image classification- a preliminary assessment," Med. Eng. \& Phys., vol. 33(8), pp. 1017-1026, 2011.

\section{AUTHORS}

Dror Lederman, PhD, BEMS, LLB

Faculty of Electrical Engineering

Holon Institute of Technology, Holon, Israel

\section{Short Biography}

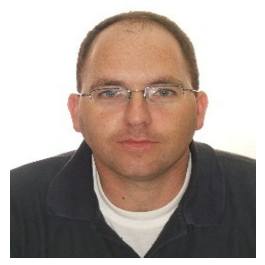

Dr. Dror Lederman received the B.Sc., M.Sc., and Ph.D. degrees in Electrical Engineering from Ben Gurion University of the Negev, Beer Sheva, Israel in 1998, 2003 and 2009, respectively, the B.EMS. in Emergency Medicine from Ben Gurion University of the Negev, Beer Sheva, Israel in 2005, and the LL.B. degree in Law from Tel-Aviv University, Tel-Aviv, Israel. He is currently a senior lecturer at the Holon Institute of Technology. His research interests include mainly machine learning, artificial intelligence and computer-aided diagnosis. Dr. Lederman also serves as a paramedic in the Red Cross Chapter in Israel ("Magen David Adom"). 